\title{
Inosine Dialdehyde
}

National Cancer Institute

\section{Source}

National Cancer Institute. Inosine Dialdehyde. NCI Thesaurus. Code C1136.

A toxic purine analogue. Inosine dialdehyde inhibits ribonucleotide reductase, resulting in decreased synthesis of DNA, RNA, and proteins, and G2/M-phase cell cycle arrest. This agent also forms stable covalent crosslinks in proteins, thereby inhibiting the activity of enzymes involved in nucleic acid synthesis. ( $\mathrm{NCl04)}$ 